\title{
Effect of a single asenapine treatment on Fos expression in the brain catecholamine-synthesizing neurons: impact of a chronic mild stress preconditioning
}

\author{
Osacka J, Horvathova L, Majercikova Z, Kiss A
}

\author{
Institute of Experimental Endocrinology, Biomedical Research Center, Slovak Academy of Sciences, Bratislava, Slovakia \\ E-mail: alexander.kiss@savba.sk
}

Objective. Fos protein expression in catecholamine-synthesizing neurons of the substantia nigra (SN) pars compacta (SNC, A8), pars reticulata (SNR, A9), and pars lateralis (SNL), the ventral tegmental area (VTA, A10), the locus coeruleus (LC, A6) and subcoeruleus (sLC), the ventrolateral pons (PON-A5), the nucleus of the solitary tract (NTS-A2), the area postrema (AP), and the ventrolateral medulla (VLM-A1) was quantitatively evaluated after a single administration of asenapine (ASE) (designated for schizophrenia treatment) in male Wistar rats preconditioned with a chronic unpredictable variable mild stress (CMS) for 21 days. The aim of the present study was to reveal whether a single ASE treatment may 1) activate Fos expression in the brain areas selected; 2) activate tyrosine hydroxylase (TH)-synthesizing cells displaying Fos presence; and 3) be modulated by CMS preconditioning.

Methods. Control (CON), ASE, CMS, and CMS+ASE groups were used. CMS included restraint, social isolation, crowding, swimming, and cold. The ASE and CMS+ASE groups received a single dose of ASE ( $0.3 \mathrm{mg} / \mathrm{kg}$, s.c.) and CON and CMS saline (300 $\mu \mathrm{l} / \mathrm{rat}$, s.c.). The animals were sacrificed 90 min after the treatments. Fos protein and TH-labeled immunoreactive perikarya were analyzed on double labeled histological sections and enumerated on captured pictures using combined light and fluorescence microscope illumination.

Results. Saline or CMS alone did not promote Fos expression in any of the structures investigated. ASE alone or in combination with CMS elicited Fos expression in two parts of the SN (SNC, SNR) and the VTA. Aside from some cells in the central gray tegmental nuclei adjacent to LC, where a small number of Fos profiles occurred, none or negligible Fos occurrence was detected in the other structures investigated including the LC and sLC, PON-A5, NTS-A2, AP, and VLM-A1. CMS preconditioning did not influence the level of Fos induction in the SN and VTA elicited by ASE administration. Similarly, the ratio between the amount of free Fos and Fos colocalized with TH was not affected by stress preconditioning in the SNC, SNR, and the VTA.

Conclusions. The present study provides an anatomical/functional knowledge about the nature of the acute ASE treatment on the catecholamine-synthesizing neurons activity in certain brain structures and their missing interplay with the CMS preconditioning.

Key words: asenapine, chronic mild stress preconditioning, Fos immunohistochemistry, tyrosine hydroxylase immunohistochemistry, rat

Corresponding author: Dr. Alexander Kiss, PhD., D.Sci., Institute of Experimental Endocrinology, Biomedical Research Center, Slovak Academy of Sciences, Dubravska cesta 9, 84505 Bratislava, Slovak Republic; tel: 00421-2-532295225; e-mail: ueenkiss@savba.sk. 
Brain is a settlement of a number of catecholaminesynthesizing cell groups residing in different brain structures (Dahlstrom and Fuxe 1964; Bjorklund et al. 1973) and involved in many physiological and pathological processes (Hui et al. 2003; Kvetnansky et al. 2009).

The dopaminergic $\mathrm{SN}$ is a structure located in the midbrain playing an important role in the eye movement, motor planning (Parkinson's disease is characterized by the death of dopaminergic neurons in the substantia nigra), reward-seeking, learning, addiction, etc. (Burns et al. 1983; Chinta and Andersen 2005; Groger et al. 2014). Actually, SN consists of three different parts, pars compacta, pars reticulata, and pars lateralis (Dahlstrom and Fuxe 1964). The SNC serves mainly as an input to the basal ganglia circuit, supplying the striatum with dopamine, while the SNR serves mainly as an output, conveying signals from the basal ganglia to many other brain areas (Lee and Tepper 2009). The SNL is made up of different neuronal populations: one projecting to the inferior colliculus and another directed to the striatum and amygdala (Moriizumi et al. 1992).

The dopaminergic VTA, located in the bottom of the midbrain just above the interpeduncular nucleus, is origin of the frontal mesocortical dopaminergic system projecting to certain cortical and mesolimbic terminal fields, including the nucleus accumbens and olfactory tubercles, implicated in reward, pleasure, and addictive behavior (Yun et al. 2004; Ranaldi 2014; MacInnes et al. 2016). The term VTA is often used as a synonym for A10 dopaminergic cell group.

The noradrenergic LC is situated in the dorsal pontine tegmentum on the lateral sides of the fourth ventricle. It is the major noradrenergic nucleus of the brain (Berridge and Waterhouse 2003) innervating via the dorsal noradrenergic bundle many brain areas involved in the control of different physiological functions, including the regulation of arousal, the autonomic function influencing the sympathetic and parasympathetic activities, wakefulness, stress response, anxiety, and depression (Samuels and Szabadi 2008).

The noradrenergic sLC spreads ventrally to LC (Westlund and Coulter 1980). The sLC neurons are directed to the sympathetic preganglionic neurons of the intermediolateral cell column of the thoracic cord. The sLC neurons also heavily project to the cranial somatic nerve nuclei (Westlund and Coulter 1980), involved in physiological responses to stress and panic.

The pontine A5 noradrenergic cell group is strongly involved in the brain control of the cardiovascular function (Woodruff et al. 1986) and displays a clear central respiratory modulation (Guyenet et al. 1993).

The dorsomedial A2 medullary noradrenergic neurons are widespread in the NTS, i.e. in the major sensory nucleus in the dorsal medulla, receive cardiovascular, visceral, respiratory, gustatory, and orotactile signals (Chan et al. 1995; Williams et al. 2000). The adjacent area postrema (AP), a chemoreceptor trigger zone, is one of the so-called circumventricular organs located on the dorsal inferior surface of the medulla oblongata at the caudal end of the fourth ventricle (Bessing et al. 1987; Horsburgh and Massoud 2013). The blood has direct access to AP neurons because its location outside of the blood-brain barrier and thus receives inputs from blood-borne drugs or hormones. It is considered to be an integrative area for various toxic signals preventing of intoxication (Ossenkopp and Eckel 1995).

The caudal ventrolateral A1 medullary noradrenergic neurons are responding to variety of visceral, somatic, and supramedullary inputs in a manner that closely mimics the effects of these inputs on the peripheral sympathetic vasomotor outflow (Guyenet et al. 1996).

The data of antipsychotic studies regarding the catecholamines involvement are extensive. It has been shown that the effects of the antipsychotic drugs on catecholamines may be dose- or sort-dependent. Differences in the effect of the haloperidol, clozapine, olanzapine, risperidone, and ziprasidone on the extracellular levels of dopamine and noradrenaline in the medial prefrontal cortex and dopamine in the striatum have been demonstrated (Westerink et al. 2001). Conversely, noradrenaline reuptake inhibition has been shown to augment the efficacy of the classical D2-antagonists in the treatment of schizophrenia (Linner et al. 2002). The DA metabolite 3,4-dihydroxyphenylacetic acid (DOPAC) levels have been shown to be selectively increased in the VTA-A10 but not A9 region cell bodies by foot shock and conditioned stress paradigm indicating that the cell bodies of the mesocortical dopaminergic system origin are activated by stress in contrast to dopamine neurons innervating the striatum (Deutch et al. 1985).

Fos protein expression has generally been used as a cell activity marker (Dragunow and Faull 1989). In the SNC, Fos expression increase has been demonstrated after 60 min of sleep deprivation induced by gentle handling, suggesting that dopaminergic nigral activation may be triggered by sleep deprivation (SD) earlier than motor responsiveness (Santos et al. 2008). It has also been shown that the modulation of target gene expression by Fos may influence the addictive 
behavioral responses to opiates (Bontempi and Sharp 1997). Expression of Fos in transmitters-characterized neurons after stress (corticotropin-releasing factor/ catecholamine neurons/paraventricular nucleus/medulla oblongata/colchicine) has also been monitored (Ceccatelli et al. 1989). It has been demonstrated that TH neurons in the NTS may be activated during different states of arousal (Asmus and Newman 1994).

The objective of the present study was to investigate the Fos protein expression in the catecholamine-synthesizing neurons in selected brain areas including the substantia nigra, the ventral tegmental area, the locus coeruleus and subcoeruleus, the ventrolateral pons, the nucleus of the solitary tract, area postrema, and the ventrolateral and dorsomedial medulla, in non-stressed and mild stress preconditioned animals after one-shot ASE administration. The aim of the study was to reveal whether acute ASE treatment may 1) activate Fos expression in the catecholaminergic areas selected; 2) activate tyrosine hydroxylase (TH)synthesizing cells indicated by Fos protein presence; and 3) be modulated by CMS preconditioning. In this study, single and double light and fluorescence microscopic immunohistochemistry was employed and the data quantitatively evaluated $90 \mathrm{~min}$ after ASE administration.

\section{Materials and Methods}

Animals. Adult male Wistar rats $(\mathrm{n}=28$, Charles River, Germany) weighing 220-250 g were used. They were housed two per cage in a room with controlled temperature $\left(22 \pm 1^{\circ} \mathrm{C}\right)$, light (12-hour light/dark cycle with lights on at 06:00 a.m.), and humidity (55 $\pm 10 \%)$. Animals were provided with a regular rat chow (dry pellets) and tap water ad libitum. Principles of the laboratory animal care and the experimental procedures used were approved by Animal Care Committee of the Institute of Experimental Endocrinology, Biomedical Research Center, Slovak Academy of Sciences, Slovak Republic. The investigation was carried out in accordance with the National Institute of Health Guide for the Care and Use of Laboratory Animals (NIH Publications No. 80-23) revised 1996 guidelines for the Care and Use of Laboratory Animals published by the U.S. National Institutes of Health. An effort was made to minimize the number and suffering of animals.

Experimental design. The rats were divided into 4 groups: control (vehicle, $\mathrm{CON}, \mathrm{n}=6$ ), asenapine (ASE, n=6), CMS + vehicle (CMS, n=8), and CMS + asenapine (CMS+ASE, $n=8)$. The CMS consisted of a combination of different mild stressors (Majercikova et al. 2014; Majercikova and Kiss 2015, 2016), including: restraint (RE, animals were placed into plastic restrainers), social isolation (SI, animals were kept individually in cages), crowding ( $\mathrm{CR}$, animals from two cages were placed into one cage), cold (CO, animals with cages were placed into a cold room at $4{ }^{\circ} \mathrm{C}$ ), and swimming (SW, animals were put into $45 \mathrm{~cm}$ high $\times 25 \mathrm{~cm}$ wide glass cylinders, filled with normal $25 \pm 1{ }^{\circ} \mathrm{C}$ water up to $36 \mathrm{~cm}$ of the height). The animals received one stress challenge per day in the following sequence and duration of exposure: RE (from 09:00 to 09:30 a.m.) - SI (overnight) - CR (overnight) - RE (from 12:00 a.m. to 00.30 p.m.) - CO (from 09:00 to 09:30 a.m.) - SI (overnight) - CO (from 12:00 a.m. to 00.30 p.m.) - RE (from 12:00 a.m. to 00.45 p.m.) CO (from 09:00 to 09:45 p.m.) - SI (overnight) - RE (from 12:00 a.m. to 00.45 p.m.) - SW (from 09:00 to 09:15 a.m., the rats were exchanged in $15 \mathrm{~min}$ intervals) - SW (from 09:00 to 09:05 a.m., the rats were exchanged in 5 min intervals) - SI (overnight) - RE (from 12:00 a.m. to 00:30 p.m.) - CO (09:00 to 09:45 a.m.) - SE (overnight) - RE (12:00 a.m.to 01:00 p.m.) - CR (overnight) - CO (12:00 a.m. to 01:00 p.m.) - SI (overnight). In order to minimize the stressors predictability and avoiding the animals to adapt to the stress stimulus, the particular stressor was applied each day at different time.

The CMS and CMS+ASE animals were exposed to the stressor for 21 days. On the $22^{\text {nd }}$ day, ASE and CMS+ASE treated groups of rats were injected subcutaneously (s.c.) with ASE (0.3 mg/kg of b.w. dissolved in saline (Sigma St. Louis MO, A7861) and CON and CMS ones with saline (s.c., $300 \mu \mathrm{l} / \mathrm{rat}$ ), respectively.

Fos/TH immunohistochemistry. The animals were sacrificed by a transcardial perfusion with fixative under sodium pentobarbital (50 mg/kg, i.p., Spofa, Czech Republic) anesthesia 90 min after the saline or ASE injection. The perfusion was performed via the aortic arch with $50 \mathrm{ml}$ of cold isotonic saline containing $450 \mu \mathrm{l}$ of heparin (5000 IU/l, Zentiva, Slovakia) followed by $250 \mathrm{ml}$ of fixative containing $4 \%$ paraformaldehyde (Sigma-Aldrich, Germany) in $0.1 \mathrm{M}$ phosphate buffer (PB, $\mathrm{pH} 7.4)$. The brains were removed from the skulls, postfixed in a fresh fixative overnight, washed in $0.1 \mathrm{M} \mathrm{PB}$ at $4{ }^{\circ} \mathrm{C}$ overnight, and infiltrated with $30 \%$ sucrose (Slavus, Slovakia) for $48 \mathrm{~h}$. Four series of coronal sections of $30 \mu \mathrm{m}$ thickness were cut on a cryocut (Hyrax c-50). They were repeatedly washed in cold $0.1 \mathrm{M} \mathrm{PB}$ and preincubated with $0.3 \% \mathrm{H}_{2} \mathrm{O}_{2}$ (Sigma-Aldrich, Germany) in $0.1 \mathrm{M}$ $\mathrm{PB}$ for $15 \mathrm{~min}$ at room temperature (RT). Thereafter, the sections were rinsed $3 \times 10 \mathrm{~min}$ in $0.1 \mathrm{M} \mathrm{PB}$ and incubated with a rabbit anti-Fos polyclonal antibody 
(1:3000) in $0.1 \mathrm{M} \mathrm{PB}$ containing $4 \%$ normal goat serum (Gibco, Grand Island, NY, USA), 0.3\% Triton X-100 (Sigma-Aldrich, Germany), and 0.1\% sodium azide (Sigma-Aldrich, Germany) for $48 \mathrm{~h}$ at $4^{\circ} \mathrm{C}$.

Fos staining was visualized by two different ways:

a) One set of Fos sections containing SN and VTA, after several rinsing in $\mathrm{PB}$, was incubated with biotinylated goat anti-rabbit IgG (1:500) in PB for 90 min at RT. Next PB rinsing was followed by incubation with the avidin-biotin peroxidase complex (1:250) for $90 \mathrm{~min}$ at RT. After several washings in $0.05 \mathrm{M}$ sodium acetate buffer (SAB, pH 6.0), Fos-antigenic sites were visualized by $3,3^{\prime}$-diaminobenzidine tetrahydrochloride $(0.0625 \% \mathrm{DAB}$ enhanced with $2.5 \%$ nickel chloride, Sigma-Aldrich, Germany), in SAB containing $0.0006 \% \mathrm{H}_{2} \mathrm{O}_{2}$. Developing time was $6-8 \mathrm{~min}$. The heavy metal-intensification of DAB yielded to black staining in the Fos-labeled nuclei.

b) Another set of Fos sections containing LC, sLC, PON-A5 NTS-A2, AP, and VLM-A1, after several rinsing in $\mathrm{PB}$, was incubated with the goat anti-rabbit IgG labeled with Alexa Fluor555 (1:250) overnight at RT.

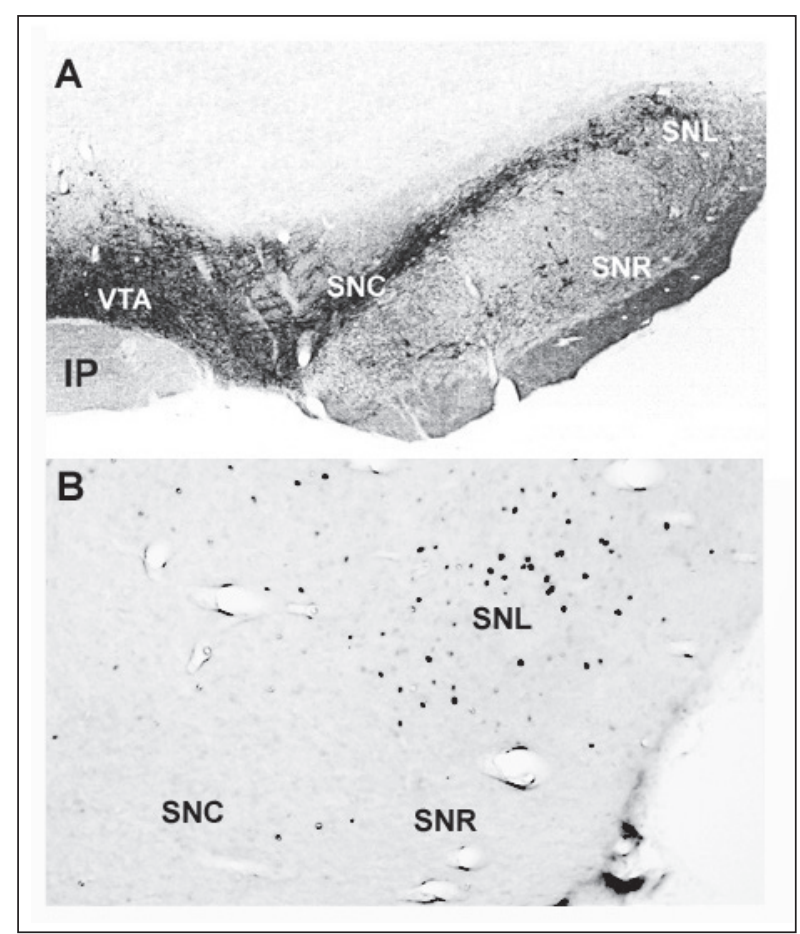

Figure 1. Distribution of TH-immunoreactive perikarya and projections in the ventral midbrain within the SNC, SNR, SNL, and VTA (A). Picture (B) demonstrates Fos profiles density and their distribution in SNL, which is representative for all four groups of animals and for all experimental conditions. Abbreviations: IP - interpeduncular nucleus, SNC - substantia nigra pars compacta SNR - substantia nigra pars reticulata, SNL substantia nigra pars lateralis, VTA - ventral tegmental area
The colocalization of Fos with TH was performed in both Fos-staining procedures by incubation of the Fos-labeled sections with the chicken anti-tyrosine hydroxylase polyclonal antibody (TH, 1:2000) labeled either with Alexa Fluor 555 labeled goat anti-chicken IgG (1:250) (a - section processing) or Alexa Fluor 488 labeled goat anti-chicken IgG (1:250) (b - section processing), both overnight at RT. Finally, the tissue sections were mounted in semi-darkness onto slides, left to dry in RT, coverslipped with Pertex (Stockholm, Sweden), and stored in dark histological boxes.

The brain structures studied. The location of the brain structures studied, including the substantia nigra pars compacta, reticularis, and lateralis, the ventral tegmental area, the locus coeruleus and subcoeruleus, the central gray tegmental nucleus, pons, the nucleus of the solitary tract, the area postrema, and the ventrolateral medulla, was identified based on the rat brain atlas (Paxinos and Watson 2007) with coordinates for SN and VTA = bregma -5.60-6.04 mm; LC, sLC, PONA5 = bregma $-9.68-10.04 \mathrm{~mm}$; and NTS, AP, VLM-A1 = bregma $-13.68-14.08)$. The single Fos profiles counting was performed unilaterally (in Adobe Photoshop 7.0 program) from photomicrographs captured from 5-6 representative sections/structure in an Axio-Imager A1 light microscope (Carl Zeiss, Jena) coupled to a video camera and monitor. An average count per section was determined for each animal. The identification of Fos/TH colocalizations was performed on photomicrographs captured on the above-mentioned microscope, using double fluorescent or synchronized fluorescent and transmission illuminations, as described previously (Majercikova et al. 2012). Parallel fluorescent and transmission illumination, the transmission light served to adjust the correct ratio between the intensity of the two beams to reach final picture clearly revealing Fos-immunolabeled nuclei within the fluorescent perikarya. Actually, the Fos/TH colocalization was expressed in percentage, whereas the percentage represents the ratio between the total Fos-labeled vs. Fos/TH colocalized perikarya.

Antibodies. The primary rabbit anti-Fos antibody is a gift from Dr. J.D. Mikkelsen (Denmark). The chicken anti-TH polyclonal antibody (ab76442), the secondary goat anti-chicken antibody (ab150114), Alexa Fluor 555 labeled goat anti-rabbit IgG (ab150078), and Alexa Fluor 488 labeled goat anti-chicken IgG (ab150169) were purchased from Abcam (Cambridge, UK). The Avidin-biotin-peroxidase complex (ABC, PK-6101, VectorStain Elite ABC Kit) was obtained from Vector Lab. (Burlingame, CA, USA).

Statistical analysis. Statistical quantification of Fos expression was carried out by two-way analysis of 
variance (ANOVA) followed by Tukey's post hoc test. Statistical evaluation was performed by SIGMASTAT software for windows (StatSoft). All the data were expressed as mean \pm SEM. The value of $p<0.05$ was considered as statistically significant.

\section{Results}

Systematic mapping of the Fos protein expression was performed on the serial coronal sections, containing the selected brain catecholamine-synthesizing structures, including SNC, SNR, SNL, LC, sLC, PON-A5, NTS-A2, AP, and VLM-A1 in four groups of animals (CON, ASE, CMS, and CMS+ASE) that underwent 21 days-lasting mild stress preconditioning.

Between the individual groups of animals and brain structures investigated different amount of Fos expression occurred. Generally, in the CON and CMS groups of animals, none or only a negligible number of neurons displayed Fos expression in the catecholamine-synthesizing neurons including the SNC, SNR, and VTA, except the SNL, where regularly numerous Fos-labeled elements (25-50/section) occurred (Figures 1A, 1B) The Fos profiles were evenly distributed over the whole SNL area situated in the SNR upper margin (Figure 1B). On the other hand, no Fos expression was seen in the rest of the catecholamine-synthesizing structures investigated including LC, sLC, PON-A5 (Figure 2), AP, NTS-A2, and VLM-A1 (Figure 3).

Fos response to ASE treatment. Ninety minutes after ASE treatment, very clear Fos response was ob-
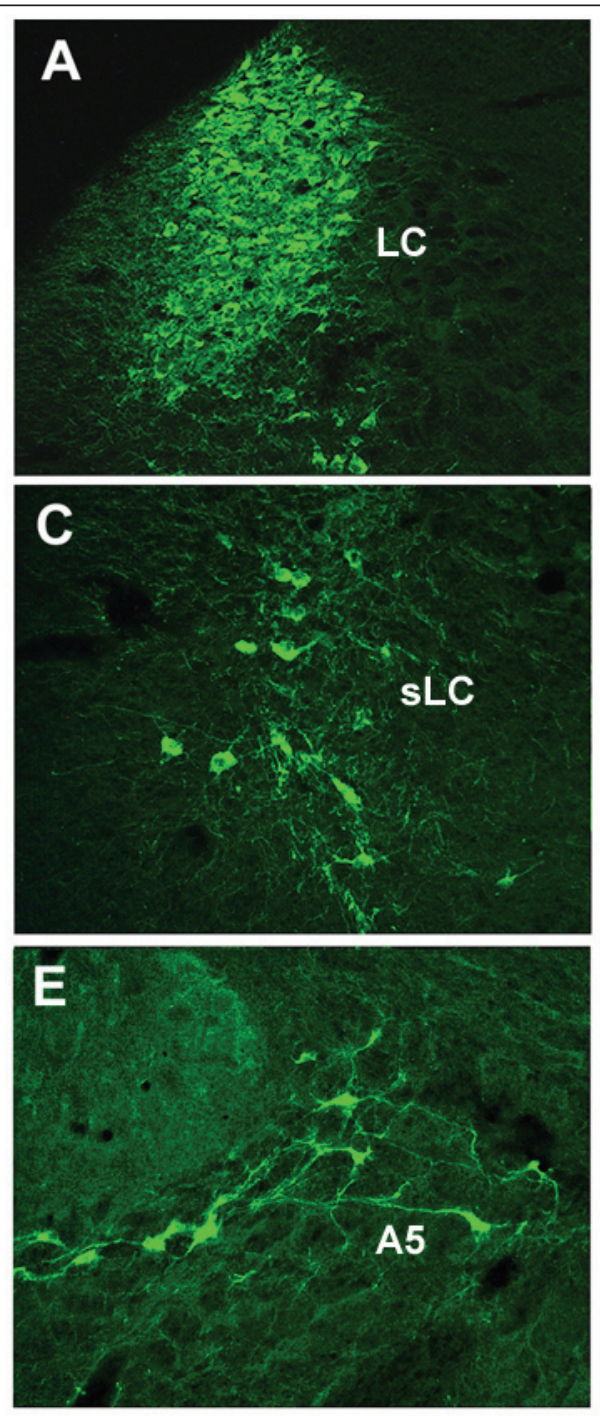
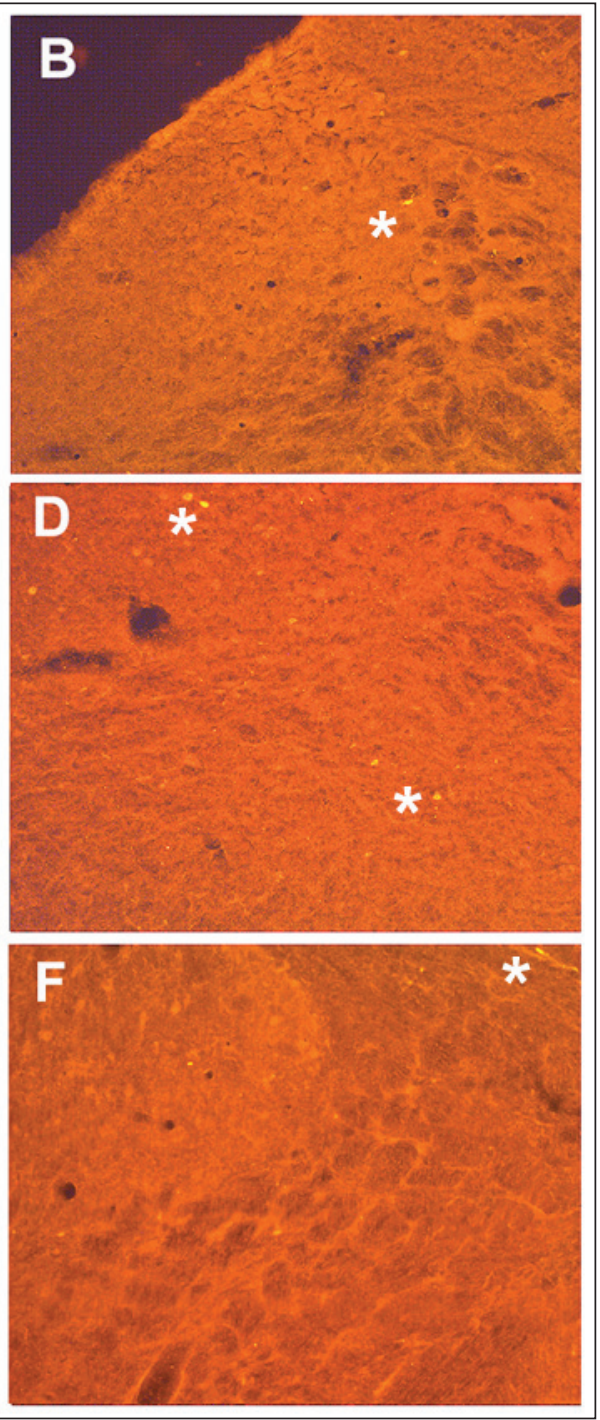

Figure 2. Effect of a single dose of ASE treatment on Fos expression in the LC $(\mathrm{A}, \mathrm{B})$, sLC $(\mathrm{C}, \mathrm{D})$, and PON-A5 area (E, F). Pictures on the left side (A, C, E) demonstrate the characteristic shape of the individual catecholamine cell group visualized by $\mathrm{TH}$ immunostaining and on the right side the response of these structures to ASE treatment. As seen, the ASE administration induced only a negligible Fos-expression (white stars) within all the three structures presented (B, D, F). Abbreviations: LC locus coeruleus, sLC - locus subcoeruleus, A5 (PON- A5) ventrolateral pons 
Figure 3. Effect of a single dose of ASE treatment on Fos expression in the NTS $(\mathrm{A}, \mathrm{B}), \mathrm{AP}(\mathrm{C}$, $\mathrm{D})$, and VLM-A1 (E, F). Pictures on the left side (A, C, E) demonstrate the characteristic shape of the individual catecholamine cell groups visualized by $\mathrm{TH}$ immunostaining and on the right side the response of these structures to ASE treatment. As seen, the ASE administration induced only a negligible Fos-expression (white stars) within all the three structures presented (B, D, F). Abbreviations: NTS - nucleus of the solitary tract (NTS-A2), AP - area postrema, A1 - ventrolateral medulla (VLM-A1)
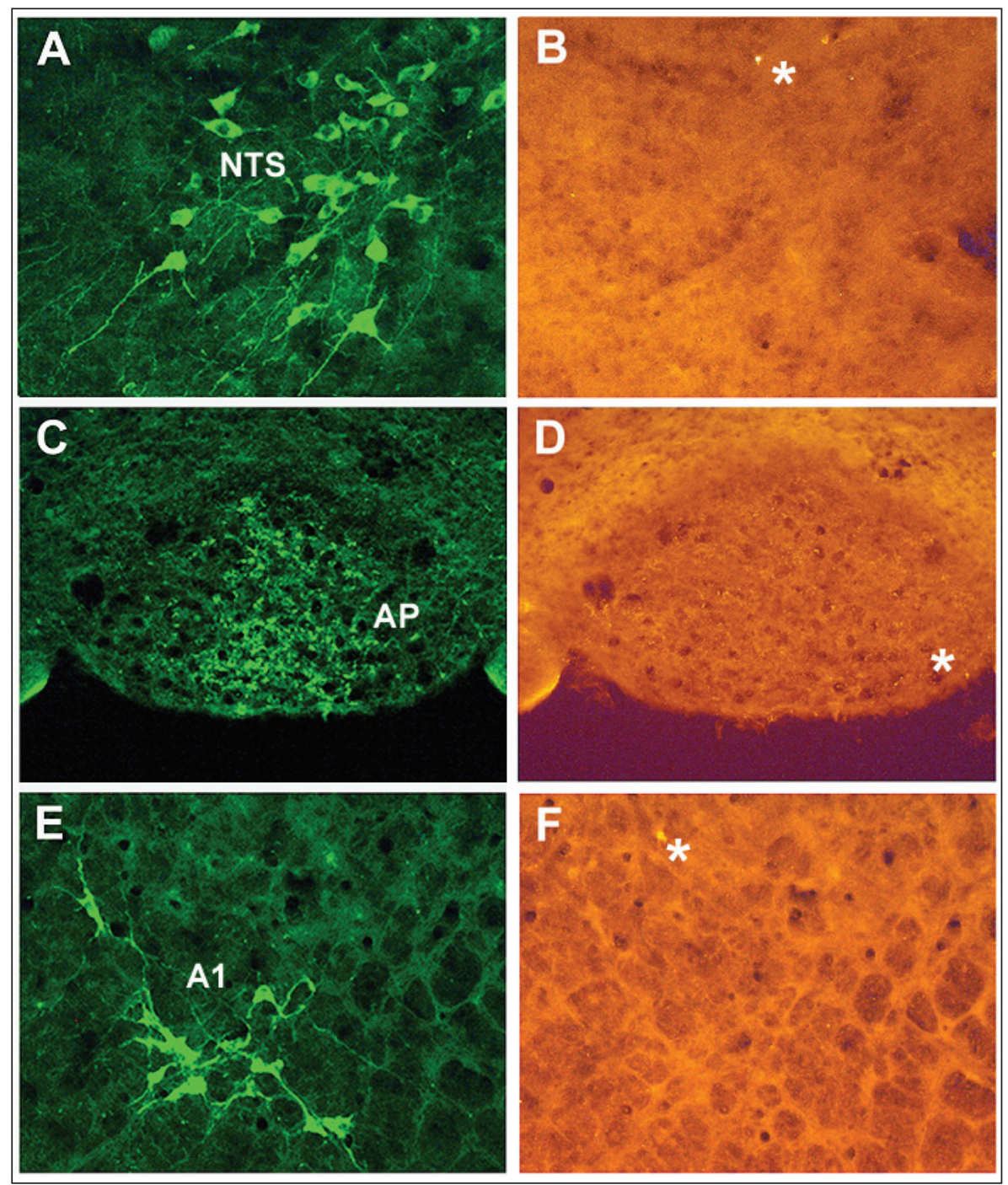

served only in the SNC, SNR, and VTA in ASE- and CMS+ASE-treated groups (Figure 4). No Fos presence was observable in ASE or CMS+ASE groups in the other catecholamine-containing structures, including LC, sLC, NTS-A2, PON-A5, AP, and VLMA1 (Figures 2, 3). Although LC neurons did not show any Fos presence, a small number of Fos-immunoreactive cell nuclei emerged in some of the adjacent central gray tegmental nuclei (not shown).

As mentioned above, distinct Fos expression was detected in the SNC, SNR, and VTA in the ASE and CMS+ASE groups of rats. Generally, Fos expression was densely-packed in the SNC and more dispersed in the SNR. In the majority ASE- and CMS+ASEtreated animals, main accumulation of Fos profiles occurred in the ventral portion of the SNC and SNR (Figure 4A). Likewise, in the SNC and SNR, the Fos profiles in the VTA were also accumulated mainly in its ventral portion adjacent to the interpeduncular nucleus (Figure 4B).

The CMS preconditioning did not affect the Fos expression in the SNL and did not significantly altered the Fos expression in the SNC, SNR, and VTA (Figure 5).

Fos/TH colocalizations. TH-immunostaining performed on the serial coronal sections revealed a large number of TH-immunolabeled cell bodies in all the brain catecholamine-containing structures selected. Most densely packed groups of TH-labeled perikarya were observed in the VTA, SNC (Figure $1 \mathrm{~A}$ ), and LC (Figure 2A). Less densely packed TH-labeled perikarya were seen in the SNR, SNL, sLC and PON-A5 (Figures 2B, 2C), NTS-A2, AP, and VLM-A1 (Figures 3A, 3B, 3C). However, Fos/TH colocaliza- 


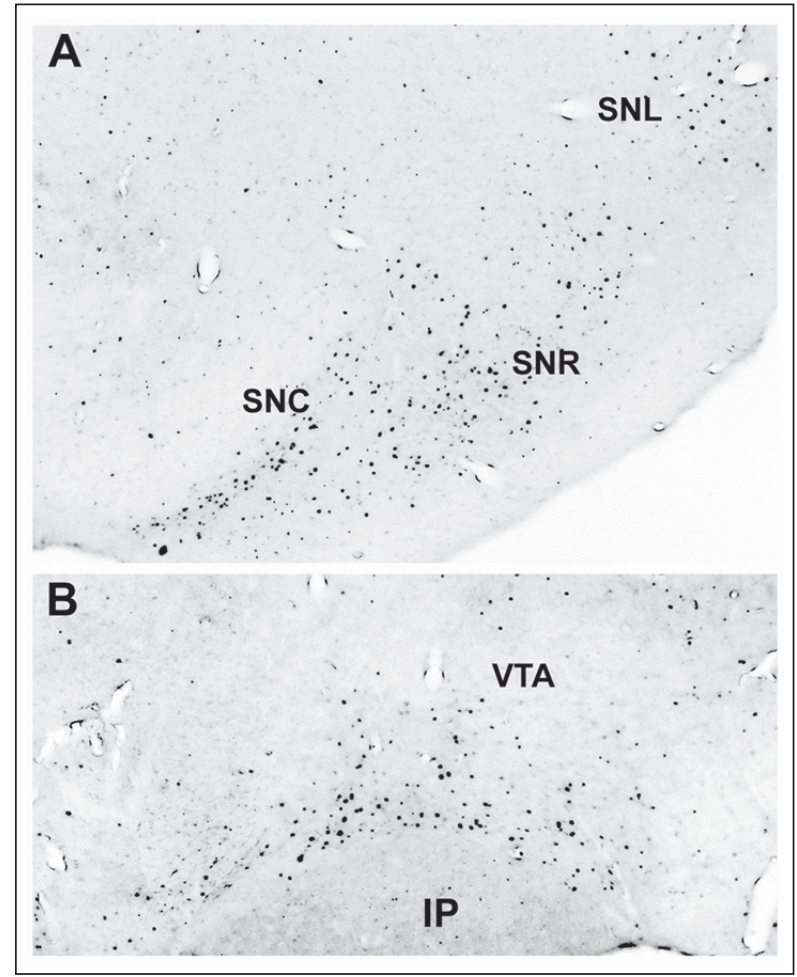

Figure 4. Representative photomicrographs demonstrating the distribution of Fos-labeled perikara after single ASE and CMS+ASE treatments in the rat SNC (A), SNR (A), and VTA (B). Fos expression in the SNL (A) did not alter by CMS preconditioning or ASE administration. Abbreviations: IP - interpeduncular nucleus, $\mathrm{SNC}$ - substantia nigra pars compacta SNR - substantia nigra pars reticulata, SNL - substantia nigra pars lateralis, VTA - ventral tegmental area

tions, visualized either by combination of two fluorescent dyes or combination of a fluorescent dye $(\mathrm{TH})$ with the nickel intensified DAB staining (Fos), were observed only in the ASE and CMS+ASE groups of animals in the SNC, SNR, and VTA. The most extensive Fos/TH colocalizations occurred in the ventral SNC, followed by the VTA and the SNR (Figure 6). In concordance with the Fos appearance, the Fos/TH colocalizations were also observable mainly in the ventral portions of the SNC, SNR, and VTA (Figure 6).

Figure 6. Fos/TH colocalizations in the SNC (A), SNR (B), and VTA $(C)$ in animal groups treated with ASE (the pictures are representative for both the ASE and CMS+ASE groups of rats). The colocalized Fos is labeled by red and free Fos by blue color. The picture was made by merging an identical TH-fluorescent and Fos-DAB-nickel stained sections and the Fos- labeled particles artificially colored in the Adobe Photoshop (7.0) channel mixer and hue/saturation programs.

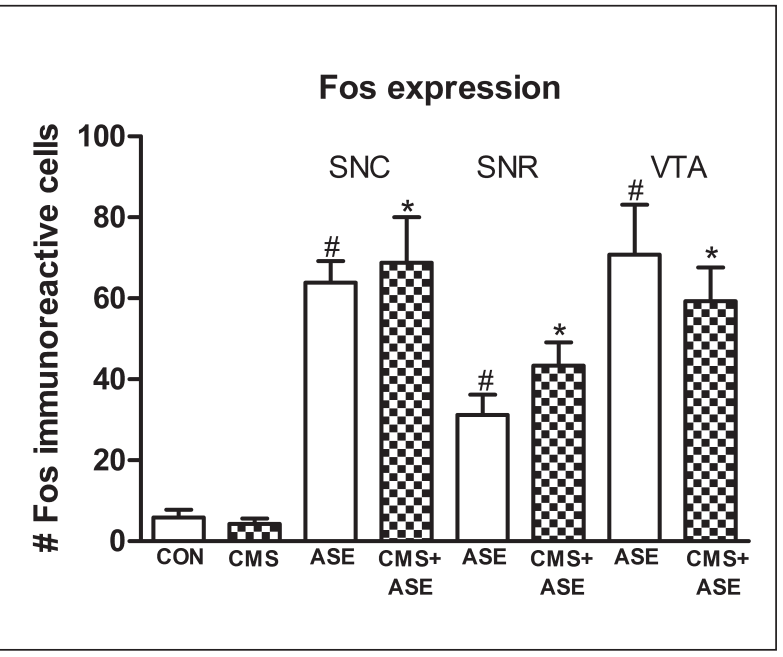

Figure 5. Fos expression response to ASE in CMS preconditioned and non-preconditioned groups of animals in the SNR, SNC, and VTA. Two-way ANOVA and Tukey's post hoc analysis revealed ASE impact on Fos-expression amount in $\operatorname{SNR}[\mathrm{F}(1,17)=57.152$, SNC $(\mathrm{F}(1,16)=59.352$, and VTA $(\mathrm{F}(1,17)=41.15$, in all $\mathrm{p}<0.001]$. Both ASE treated groups exhibited significantly more Fos profiles in all the areas studied. Comparisons in SNR = CON vs. ASE, CMS vs. CMS+ASE; in $\mathrm{SNC}=\mathrm{CON}$ vs. ASE, CMS vs. CMS+ASE; and in VTA = CON vs. ASE, CMS vs. CMS+ASE, showed statistical significance ${ }^{*} \mathrm{p}<0.001$ or $\# \mathrm{p}<0.001$. Post hoc analysis did not find interaction between the ASE and CMS+ASE groups.

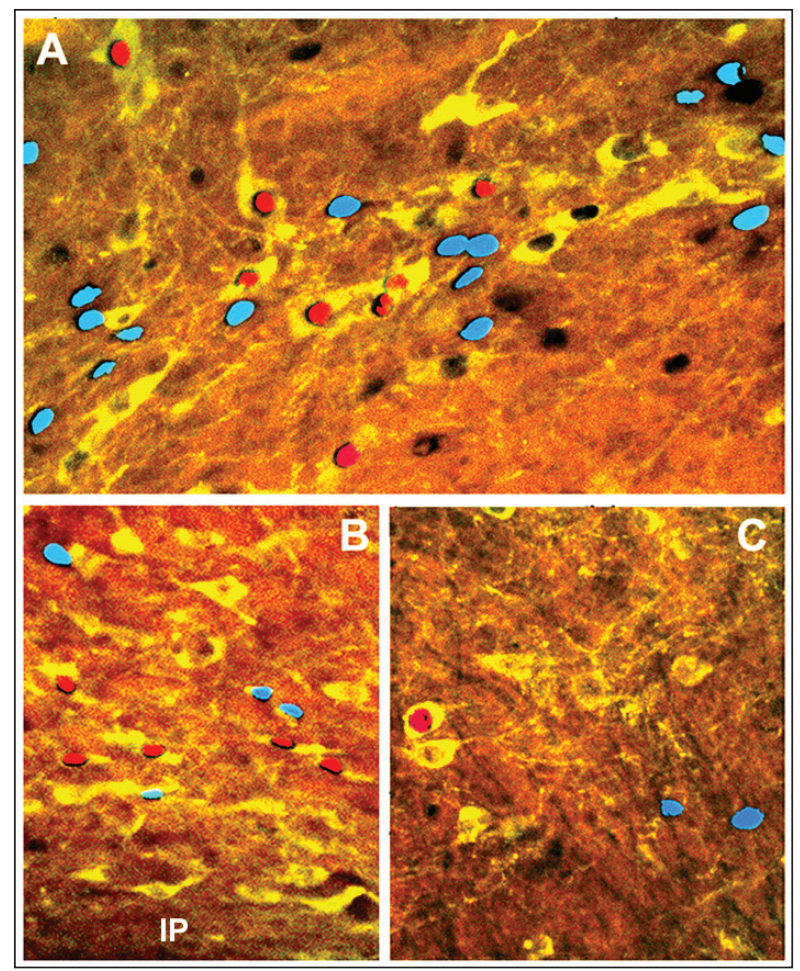




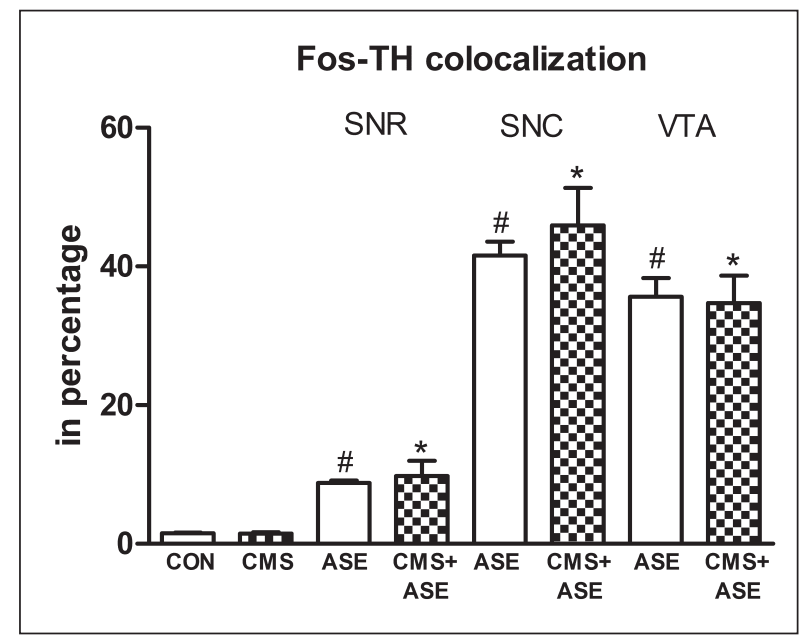

Figure 7. Level of Fos/TH colocalizations (expressed in percentage) after ASE treatment in CMS preconditioned and non-preconditioned groups of animals in the SNR, SNC, and VTA. Two-way ANOVA and Tukey's post hoc analysis revealed ASE impact on the percentage of Fos/TH co-labeled cells in the SNR $[F(1,23)=47.581, \operatorname{SNC}[F(1,23)=212.165$, and VTA $(\mathrm{F}(1,23)=198.976$, in all $\mathrm{p}<0.001]$. Both ASE-treated groups exhibited significantly more Fos profiles in TH-labeled cells. Comparisons in SNR = CON vs. ASE, CMS vs. CMS+ASE; in $\mathrm{SNC}=\mathrm{CON}$ vs. ASE, CMS vs. CMS+ASE; and in VTA = CON vs. ASE, CMS vs. CMS+ASE, showed statistical significance ${ }^{*} \mathrm{p}<0.001$ or $\# \mathrm{p}<0.001$. Post hoc analysis did not find interaction between the ASE and CMS+ASE groups.

The CMS preconditioning did not alter the number of Fos/TH-coexpressing neurons in the SNC, SNR, and VTA in CMS+ASE group of animals (Figure 7).

\section{Discussion}

The data of the present study showed that saline or CMS alone did promote only a negligible Fos expression in the brain catechomaninergic structures studied. On the other hand, ASE treatment elicited Fosexpression, however, only in the SN and the VTA. CMS preconditioning did not alter the Fos response to ASE in the SNC, SNR, and VTA. Except the central gray tegmental nuclei adjacent to LC, where a small number of Fos profiles was detectable, no Fos presence was observed in the rest of the structures investigated including LC, sLC, NTS-A2, PON-A5, AP, and VLMA1. In the SNC, SNR, and VTA of ASE and CMS+ASE groups, increased Fos/TH colocalizations from 10 to $45 \%$ occurred. The amount of Fos/TH colocalizations was not influenced by CMS preconditioning.

Catecholamine-synthesizing neurons are located in specific and anatomically well-defined brain structures, as revealed by immunohistochemical studies
(Dahlstrom and Fuxe 1964; Bjorklund and Nobin 1973; Swanson and Hartman 1975; Hokfelt et al. 1976; Lindvall and Bjorkland 1983). It has been shown that they are involved in a number of brain functions (Kobayashi 2001; Pacak and Palkovits 2001; Kvetnansky et al. 2009). The central noradrenergic pathways activation by atypical antipsychotics has been believed to play a positive role in the treating the negative symptoms and cognitive impairment of schizophrenia (Dawe et al. 2001). Using immunohistochemistry, Fos expression in the LC and sLC, but not PON-A5 area, has been induced by acute olanzapine and clozapine treatments (Dawe et al. 2001). LC is the major brain noradrenergic nucleus, influencing the activity of the entire brain via dorsal and ventral bundles of axons (Ungerstedt 1971). LC has long been thought to be involved in states of cognitive processes. These functional characteristics make this nucleus interesting with respect to the signs of schizophrenia, especially the disease negative symptoms (Nilsson et al. 2005). Ohashi et al. (2000) have even shown that clozapine and olanzapine administrations induce not only Fos expression in the LC but also noradrenaline release by stimulating noradrenergic neuronal activity in the LC, which leads to a consequent, increase of the noradrenaline induced Fos expression in the mPFC via $\beta$-adrenergic receptors. In the present study, the acute ASE treatment failed to induce Fos expression in the LC and sLC. In addition, no Fos expresion response to ASE was found in several other brain stem structures containing catecholamine-containing cells including the PON-A5, NTS-A2, AP, and VLM-A1 areas.

It is interesting to note that except the SN and VTA, none of the other structures studied was affected by ASE treatment. Thor and Helke (1988) have demonstrated that most of the VTA groups of projecting neurons are considerably intermixed with the exception of those that project to the lateral septum, the lateral habenula, and the hippocampal formation, which are concentrated in the ventral and medial parts of the VTA. In the present study, the most pronounced effect of ASE treatment on the Fos expression was detectable mainly in the ventral parts of both $\mathrm{SN}$ and VTA, which may offer a suggestion that ASE might activate certain group of neurons that project to the lateral septum, lateral habenula, and hippocampus. Indeed, previously we have demonstrated a strong stimulatory effect of a single ASE treatment on the septal neurons located in its ventral-lateral portion (Majercikova et al. 2014). Inhibition of the central dopamine functions is an important property of antipsychotic drugs, whereas the mesolimbic and nigrostriatal portions of the dopaminergic system are the main 
targets for the mental and extrapyramidal actions, respectively. Furthermore, it has been shown that injection of the D2 receptor antagonists, haloperidol and metoclopramide, in rats resulted in a dose-dependent induction of Fos-like-immunoreactivity in the medial portion of the SNR. However, the above-mentioned SNR staining occurred exclusively in neurons, which were not immunoreactive for TH and could be antagonized by pretreatment with the anticholinergic drug scopolamine (Wirtshafter and Asin 1995). In the present study, many $\mathrm{TH}$ cells were activated by the ASE not only in the SNC and VTA but also in the SNR portion, i.e. area similarly localized to that mentioned above. Actually, the phenotype character of the other populations of the activated SNR neurons, represented by only the presence of Fos-immunolabeled nuclei, was not identified in the present study.

There is no doubt that Fos expression detection is one of the most commonly used indicators of an increased cell activity in the brain induced by a number of various stimuli including stress paradigms, whereas, generally, the neuronal activation mediated by Fos levels can be detected within $0.5 \mathrm{~h}$ with the peak in 1-2 h (Morgan and Curran 1991). Different response to stressors has been shown in the mesencephalon dopaminergic cells in the case of the footshock and the conditioned stress paradigm. Both these stress conditions selectively affected the DOPAC levels by increasing them in the A10 cell area (VTA) but not in the A9 (SNR) region (Deutch et al. 1985). These data demonstrate that the cell bodies of the mesocortical dopaminergic system origin are activated by stress in contrast to DA neurons innervating the striatum, indicating that the mesocortical dopaminergic neurons exhibit different regulatory features than mesolimbic or nigrostriatal neurons (Deutch et al. 1985). Similarly, selective increases in Fos protein expression in response to stressor has been reported in the ventral mesencephalon, where restraint stress for $30 \mathrm{~min}$ increased the number of DA neurons exhibiting Foslike immunoreactivity in the ventral tegmental area (VTA), but not in the substantia nigra or retrorubral field (Deutch et al. 1991) In the present study, however, the 21 days lasting CMS preconditioning alone had no significant effect on the Fos expression in the SNC, SNR, and VTA, which might be explained by the possible adaptation of animals to the daily repeat- ed mild disturbances, since increased Fos expression has been reported in the SNC of adult male C57/BL6J mice after a gentle handling used for the introducing of a sleep deprivation (Santos et al. 2008).

On the other hand, in the present study, ASE had a strong effect on the Fos expression in the SNC, SNR, and VTA, which was not affected by the CMS. However, previously we have shown that CMS may alter the ASE-induced Fos expression in the Meynert's nucleus (Majercikova and Kiss 2016), which support the notion that different brain structures may have a different sensitivity to the same stressor. ASE treatment also induced a number of Fos/TH colocalizations in the SNC, SNR, and VTA localized mainly in the ventral positions of the mentioned structures. The exposure of female California mice to three episodes of defeat, but not a single one, induced also more Fos/ TH-positive cells in the ventral than the dorsal VTA indicating that VTA dopamine neurons in particular are more sensitive to aversive contexts compared with controls (Greenberg et al. 2015). It has also been demonstrated by activation of Fos expression that repeated social stress episodes induce a long-lasting neural change that may lead to an augmented functional activation in the VTA neurons, however the authors did not mention whether this activation also concerned the TH population of neurons.

In summary, the present data indicate that ASE does not have ubiquitous effect over the brain catecholaminergic structures, but its action is mainly oriented to the SN and VTA partially affecting the THsynthesizing neurons. In these structures, the ASE stimulatory effect is not altered by the CMS preconditioning. Thus, the present study provides a new anatomical/functional knowledge about the nature of the acute ASE treatment on the activity of certain groups of the brain catecholamine-synthesizing neurons and its missing interplay with the CMS preconditioning.

\section{Acknowledgement}

The authors are grateful for the Fos antibody (No 94012) supplied by Dr. Jens D. Mikkelsen (Denmark) and financial support provided by VEGA (2/0031/16) grant of the Slovak Academy of Sciences and APVV15-0037 grant of the Research and Development Agency of the Slovak Republic.

\section{References}

Asmus SE, Newman SW. Colocalization of tyrosine hydroxylase and Fos in the male Syrian hamster brain following different states of arousal. J Neurobiol 25, 156-168, 1994. 
Berridge CW, Waterhouse BD. The locus coeruleus-noradrenergic system: modulation of behavioral state and statedependent cognitive processes. Brain Res Rev 42, 33-84, 2003.

Bjorklund A, Nobin A. Fluorescence histochemical and microspectrofluorometric mapping of dopamine and noradrenaline cell groups in the rat diencephalon. Brain Res 51, 193-205, 1973.

Blessing WW, Hedger SC, Joh TH, Willoughby JO. Neurons in the area postrema are the only catecholamine-synthesizing cells in the medulla or pons with projections to the rostral ventrolateral medulla (C1-area) in the rabbit. Brain Res 419, 336-340, 1987.

Bontempi B, Sharp FR. Systemic morphine-induced Fos protein in the rat striatum and nucleus accumbens is regulated by $m$ opioid receptors in the substantia nigra and ventral tegmental area. J Neurosci 17, 8596-8612, 1997.

Burns RS, Chiueh CC, Markey SP, Ebert MH, Jacobowitz DM, Kopin IJ. Primate model of parkinsonism: Selective destruction of dopaminergic neurons in the pars compacta of the substantia nigra by N-methyl-4-phenyl1,2,3,6-tetrahydropyridine. Proc Natl Acad Sci USA 80, 4546-4550, 1983.

Ceccatelli S, Villar MJ, Goldstein M, Hokfelt T. Expression of c-Fos immunoreactivity in transmitter-characterized neurons after stress (corticotropin-releasing factor/catecholamine neurons/paraventricular nucleus/medulla oblongata/colchicine). Proc Natl Acad Sci USA 86, 9569-9573, 1989.

Chan JYH, Tsou MY, Len WB, Lee TY, Chan SHH. Participation of noradrenergic neurotransmission in the enhancement of baroreceptor reflex response by substance $\mathrm{P}$ at the nucleus tractus solitarii of the rat: A reverse microdialysis study. J Neurochem 64, 2644-2652, 1995.

Chinta SJ, Andersen JK. Dopaminergic neurons. Int J Biochem Cell Biol 37, 942-946, 2005.

Dahlstrom A, Fuxe K. Existence of monoamine-containing neurons in the central nervous system. I. Demonstration of monoamines in the cell bodies of brain stem neurons. Acta Physiol Scand Suppl 4, 232, 1-55, 1964.

Dawe GS, Huff KD, Vandergriff JL, Sharp T, O’Neill MJ, Rasmussen K. Olanzapine activates the rat locus coeruleus: in vivo electrophysiology and c-Fos immunoreactivity. Biol Psychiatry 50, 510-520, 2001.

Deutch AY, Tam SY, Roth RH. Footshock and conditioned stress increase 3,4-dihydroxyphenylacetic acid (DOPAC) in the ventral tegmental area but not substantia nigra. Brain Res 333, 143-146, 1985.

Deutch AY, Lee MC, Gillham MH, Cameron DA, Goldstein M, Iadarola MJ. Stress selectively increases fos protein in dopamine neurons innervating the prefrontal cortex. Cereb Cortex 1, 273-292, 1991.

Dragunow M, Faull R. The use of c-fos as a metabolic marker in neuronal pathway tracing. J Neurosci Methods 29, 261-265, 1989.

Greenberg GD, Steinman MQ, Doig IE, Hao R, Trainor BC. Effects of social defeat on dopamine neurons in the ventral tegmental area in male and female California mice. Eur J Neurosci 42, 3081-3094, 2015.

Groger A, Kolb R, Schafer R, Klose U. Dopamine reduction in the substantia nigra of Parkinson's disease patients confirmed by in vivo magnetic resonance spectroscopic imaging. PLoS ONE 9, e84081, 2014.

Guyenet PG, Koshiya N, Huangfu D, Verberne AJ, Riley TA. Central respiratory control of A5 and A6 pontine noradrenergic neurons. Am J Physiol 264, R1035-R1044, 1993.

Guyenet PG, Koshiya N, Huangfu D, Baraban SC, Stornetta RL, Li YW. Role of medulla oblongata in generation of sympathetic and vagal outflows. Prog Brain Res 107,127-144, 1996.

Hui AS, Striet JB, Gudelsky G, Soukhova GK, Gozal E, Beitner-Johnson D, Guo SZ, Sachleben LR Jr, Haycock JW, Gozal D, Czyzyk-Krzeska MF. Regulation of catecholamines by sustained and intermittent hypoxia in neuroendocrine cells and sympathetic neurons. Hypertension 42, 1130-1136, 2003.

Hokfelt T, Johansson O, Fuxe K, Goldstein M, Park D. Immunohistochemical studies on the localization and distribution of monoamine neuron systems in the rat brain. I. Tyrosine hydroxylase in the mes- and diencephalon. Med Biol 54, 427-453, 1976.

Horsburgh A, Massoud TF. The circumventricular organs of the brain: conspicuity on clinical 3T MRI and a review of functional anatomy. Surg Radiol Anat 35, 343-349, 2013.

Kvetnansky R, Sabban EL, Palkovits M. Catecholaminergic systems in stress: structural and molecular genetic approaches. Physiol Rev 89, 535-606, 2009.

Kobayashi K. Role of catecholamine signaling in brain and nervous system functions: new insights from mouse molecular genetic study. J Investig Dermatol Symp Proc 6, 115-121, 2001.

Lee CR, Tepper JM. Basal ganglia control of substantia nigra dopaminergic neurons. J Neural Transm Suppl 73, 71-90, 2009.

Lindvall O, Bjorklund A. Dopamine- and Norepinephrine-Containing Neuron Systems: Their Anatomy in the Rat Brain. In: Emson PC (Ed), Chemical Neuroanatomy, Raven Press, New York, 229-256, 1983.

Linner L, Wiker C, Wadenberg ML, Schalling M, Svensson TH. Noradrenaline reuptake inhibition enhances the antipsychotic-like effect of raclopride and potentiates D2-blockage-induced dopamine release in the medial prefrontal cortex of the rat. Neuropsychopharmacology 27, 691-698, 2002. 
MacInnes JJ, Dickerson KC, Chen N, Adcock RA. Cognitive neurostimulation: learning to volitionally sustain ventral tegmental area activation. Neuron 89, 1331-1342, 2016.

Majercikova Z, van Weering H, Scsukova S, Mikkelsen JD, Kiss A. A new approach of light microscopic immunohistochemical triple-staining: combination of Fos labeling with diaminobenzidine-nickel and neuropeptides labeled with Alexa488 and Alexa555 fluorescent dyes. Endocr Regul 46, 217-223, 2012.

Majercikova Z, Cernackova A, Horvathova L, Osacka J, Pecenak J, Kiss A. Effect of acute asenapine treatment on Fos expression in frontal brain structures under normal conditions and mild stress preconditioning in rat. Brain Res Bull 108, 60-66, 2014.

Majercikova Z, Kiss A. Stress alters asenapine-induced Fos expression in the Meynert's nucleus: response of adjacent hypocretin and melanin-concentrating hormone neurons in rat. Neurol Res 38, 32-39, 2016.

Morgan JI, Curran T. Stimulus-transcription coupling in the nervous system: involvement of the inducible protooncogenes fos and jun. Annu Rev Neurosci 14, 421-451, 1991.

Moriizumi T, Leduc-Cross B, Wu JY, Hattori T. Separate neuronal populations of the rat substantia nigra pars lateralis with distinct projection sites and transmitter phenotypes. Neuroscience 46, 711-720, 1992.

Nikulina EM, Covington HE 3rd, Ganschow L, Hammer RP Jr, Miczek KA. Long-term behavioral and neuronal cross-sensitization to amphetamine induced by repeated brief social defeat stress: Fos in the ventral tegmental area and amygdala. Neuroscience 123, 857-865, 2004.

Nilsson LK, Schwieler L, Engberg G, Linderholm KR, Erhardt S. Activation of noradrenergic locus coeruleus neurons by clozapine and haloperidol: involvement of glutamatergic mechanisms. Int J Neuropsychopharmacol 8, 329-339, 2005.

Ohashi K, Hamamura T, Lee Y, Fujiwara Y, Suzuki H, Kuroda S. Clozapine- and olanzapine-induced Fos expression in the rat medial prefrontal cortex is mediated by $\beta$-adrenoceptors. Neuropsychopharmacology 23, 162-169, 2000.

Ossenkopp KP, Eckel LA. Toxin-induced conditioned changes in taste reactivity and the role of the chemosensitive area postrema. Neurosci Biobehav Rev 19, 99-108, 1995.

Pacak K, Palkovits M. Stressor specificity of central neuroendocrine responses: implications for stress-related disorders. Endocr Rev 22, 502-548, 2001.

Paxinos G, Watson C. The Rat Brain in Stereotaxic coordinates, compact 6th ed. Academic Press, Sydney 2007.

Ranaldi R. Dopamine and reward seeking: the role of ventral tegmental area. Rev Neurosci 25, 621-630, 2014.

Samuels ER, Szabadi E. Functional neuroanatomy of the noradrenergic locus coeruleus: Its roles in the regulation of arousal and autonomic function part II: Physiological and pharmacological manipulations and pathological alterations of locus coeruleus activity in humans. Cur Neuropharmacol 6, 254-285, 2008.

Santos CA, Andersen ML, Lima MMS, Tufik S. Gentle handling temporarily increases c-Fos in the substantia nigra pars compacta. Braz J Med Biol Res 41, 920-925, 2008.

Swanson LW, Hartman BK. The central adrenergic system. An immunofluorescence study of the location of cell bodies and their efferent connections in the rat utilizing dopamine-beta-hydroxylase as a marker. J Comp Neurol 163, 467-505, 1975.

Thor KB, Helke CJ. Catecholamine-synthesizing neuronal projections to the nucleus tractus solitarii of the rat. J Comp Neurol 268, 264-280, 1988.

Ungerstedt U. Stereotaxic mapping of the monoamine pathways in the rat brain. Acta Physiologica 82, 1-48, 1971.

Westerink BH, Kawahara Y, De Boer P, Geels C, De Vries JB, Wikstrom HV, Van Kalkeren A, Van Vliet B, Kruse CG, Long SK. Antipsychotic drugs classified by their effects on the release of dopamine and noradrenaline in the prefrontal cortex and striatum. Eur J Pharmacol 412, 127-138, 2001.

Westlund KN, Coulter JD. Descending projections of the locus coeruleus and subcoeruleus/medial parabrachial nuclei in monkey: axonal transport studies and dopamine-beta-hydroxylase immunocytochemistry. Brain Res 2, 235-264, 1980.

Williams CL, Men D, Clayton EC. The effects of noradrenergic activation of the nucleus tractus solitarius on memory and in potentiating norepinephrine release in the amygdala. Behav Neurosci 114, 1131-1144, 2000.

Wirtshafter D, Asin K. Dopamine antagonists induce Fos-like-immunoreactivity in the substantia nigra and entopeduncular nucleus of the rat. Brain Res 670, 205-214, 1995.

Woodruff ML, Baisden RH, Whittington DL, Kelly JE. Inputs to the pontine A5 noradrenergic cell group: a horseradish peroxidase study. Exp Neurol 94, 782-787, 1986.

Yun IA, Wakabayashi KT, Fields HL, Nicola SM. The ventral tegmental area is required for the behavioral and nucleus accumbens neuronal firing responses to incentive cues. J Neurosci 4, 2923-2933, 2004. 